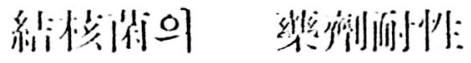

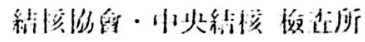

金

\section{I 緒詇}

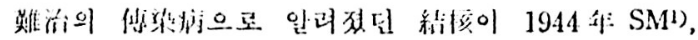

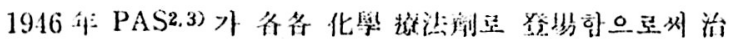

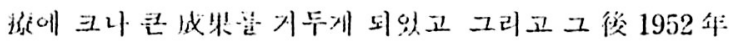
에는 INH4)가 2 ij

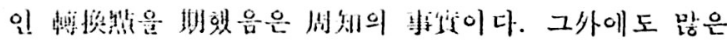

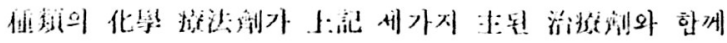

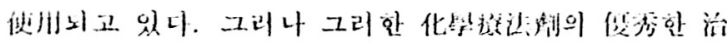

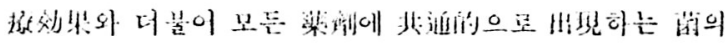

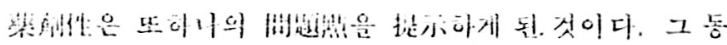

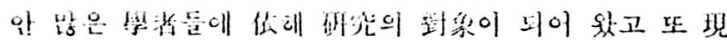

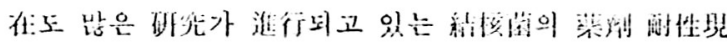

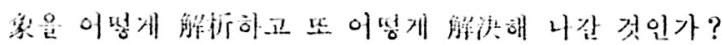

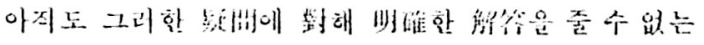

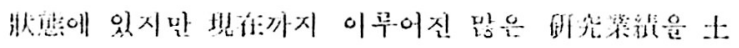

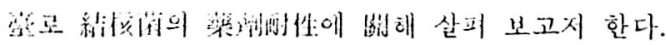

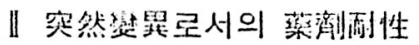

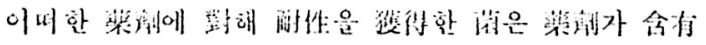

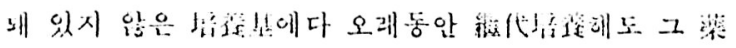

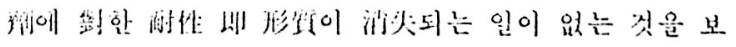

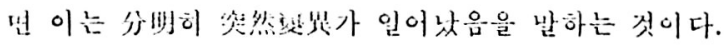

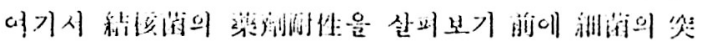

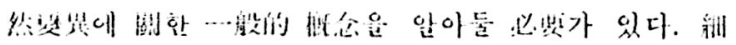

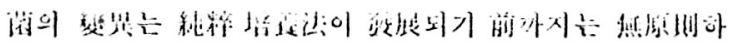

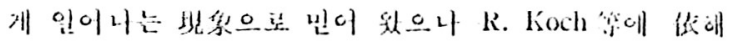

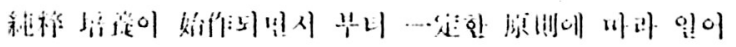

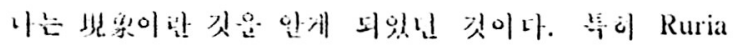

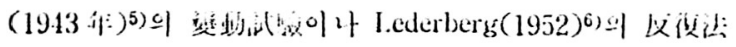

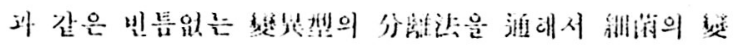

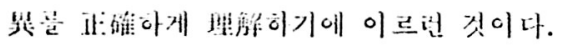

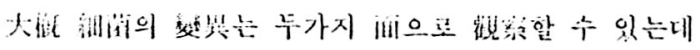

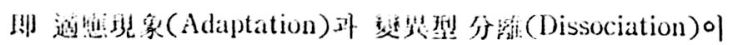

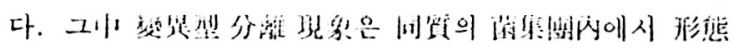

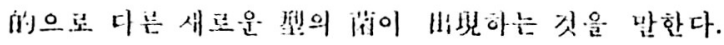

份

\section{材}

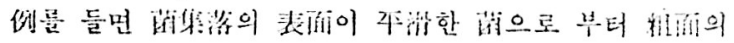

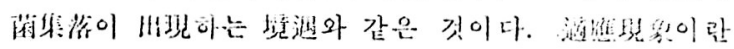

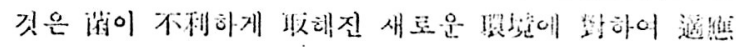

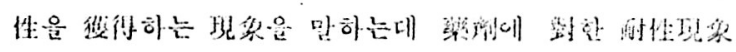

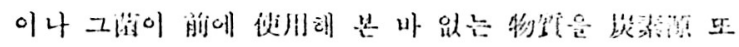

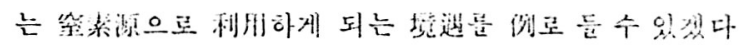

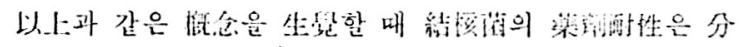

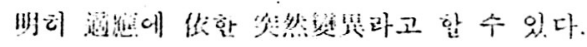

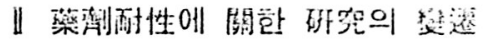

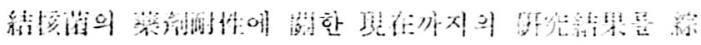

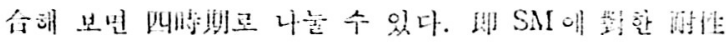

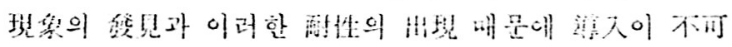

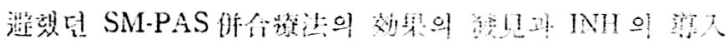

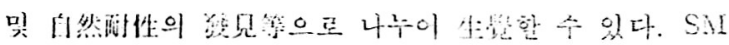

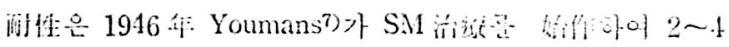

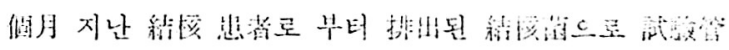

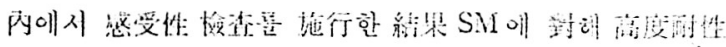

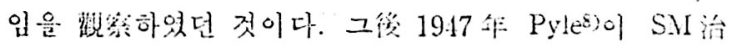

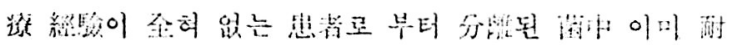

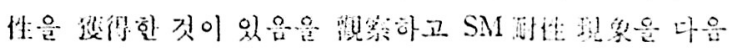

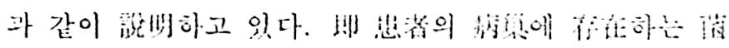

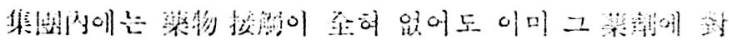

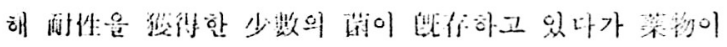

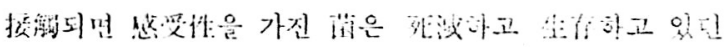

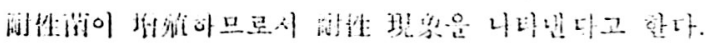

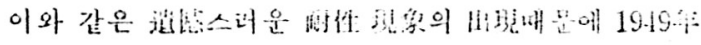

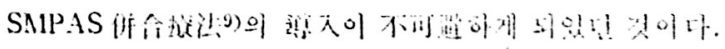

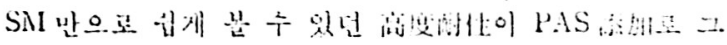

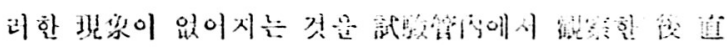

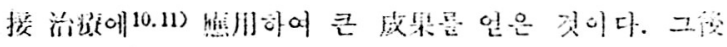

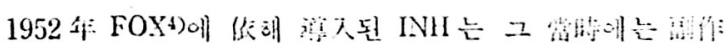

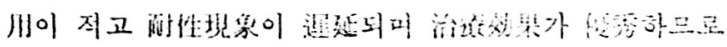

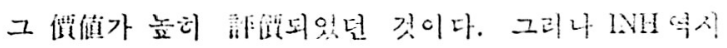

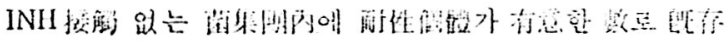

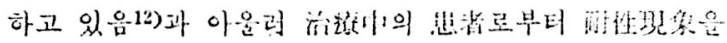




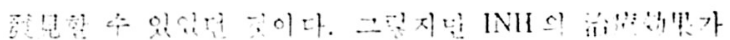

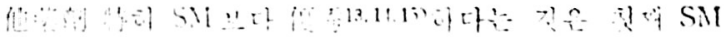
배

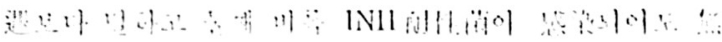

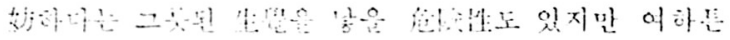

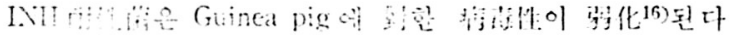
$\therefore$ पी

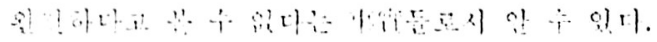

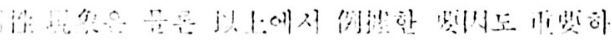

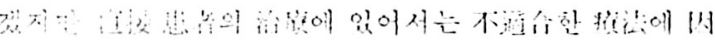

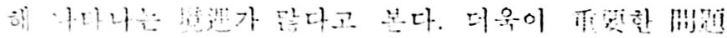

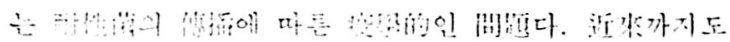

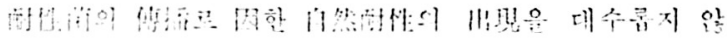

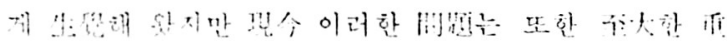

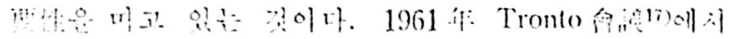

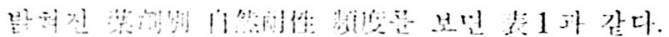

i

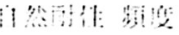

Parisii) Students Tannier ont (all Farisltics) Pationts (adults)

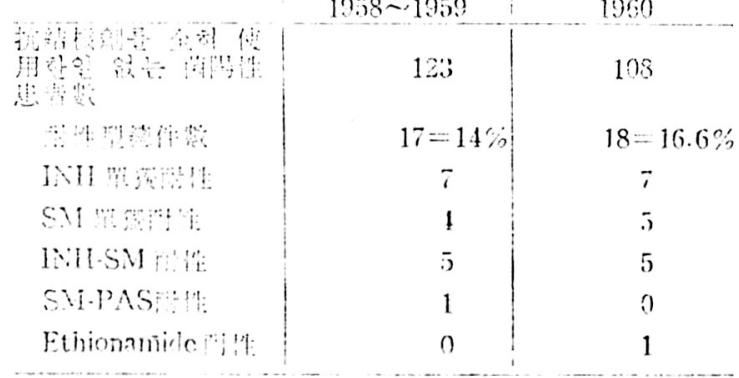

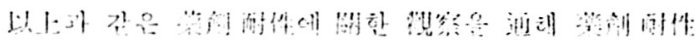

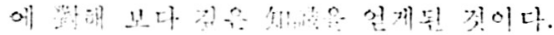

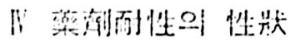

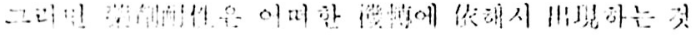

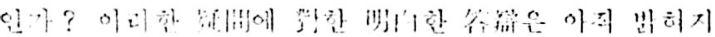
r) $2 \frac{1}{5} 3-5$.

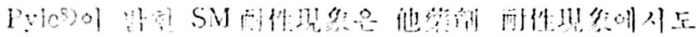

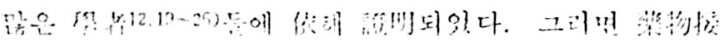

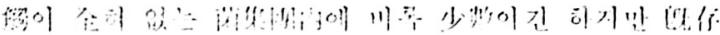

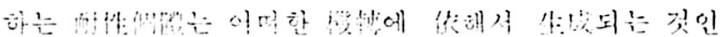

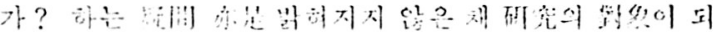
어 이는 기눈.

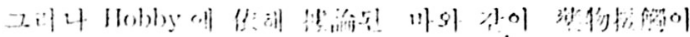

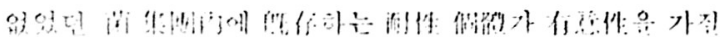

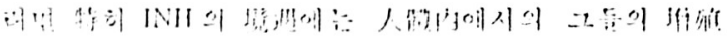

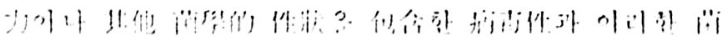

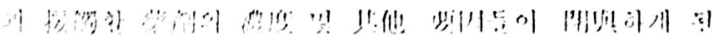

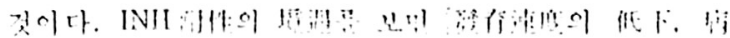

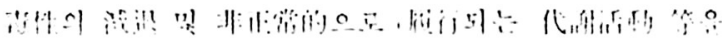

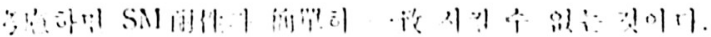

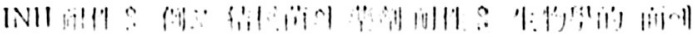

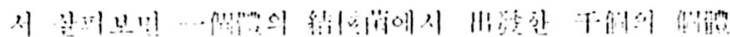

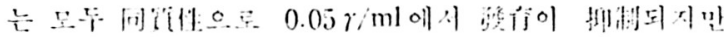

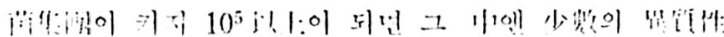

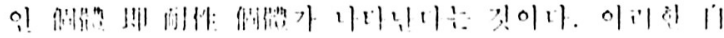

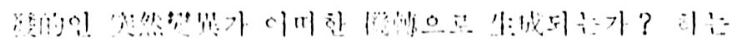

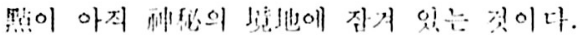

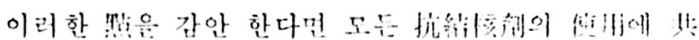

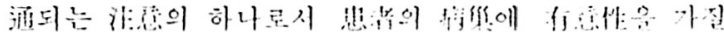

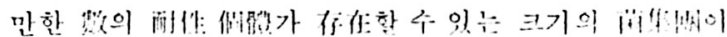

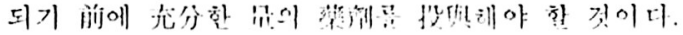

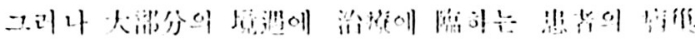

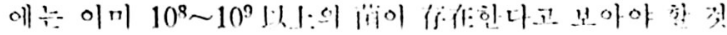

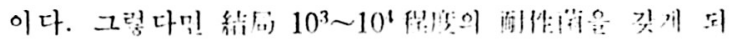

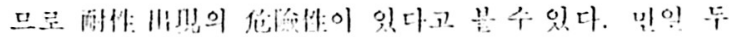

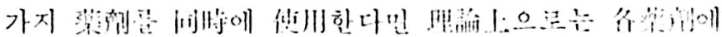

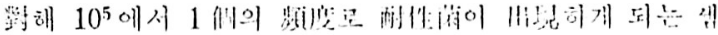

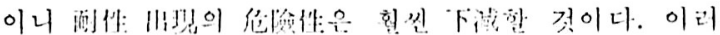

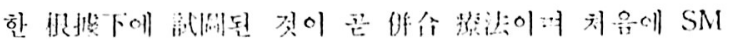

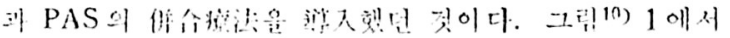

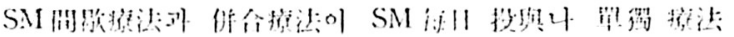

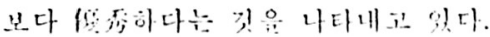

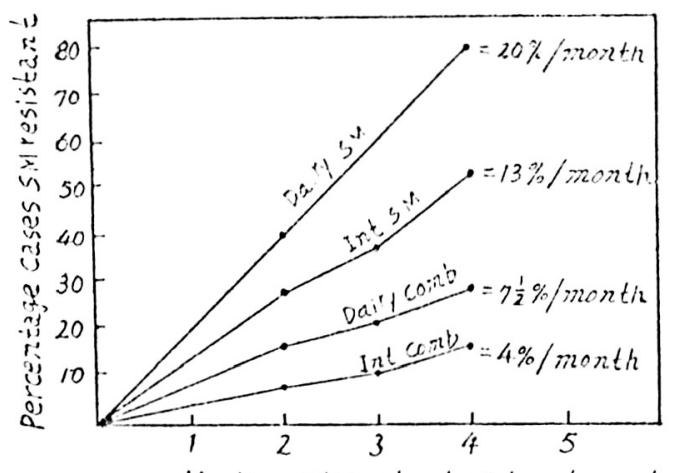

Months aiter start of treatment

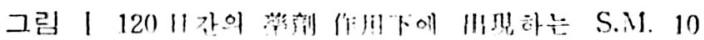

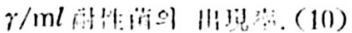

I)aily SM: SM 1 g o : fill fly

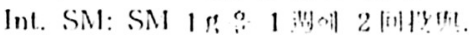

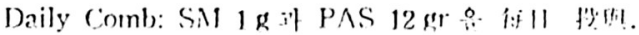

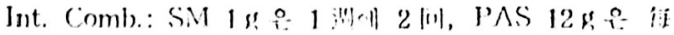
11 f'thl.

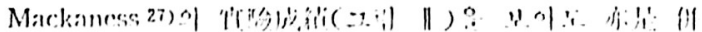




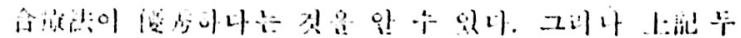

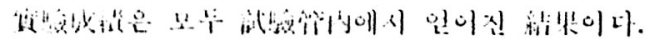

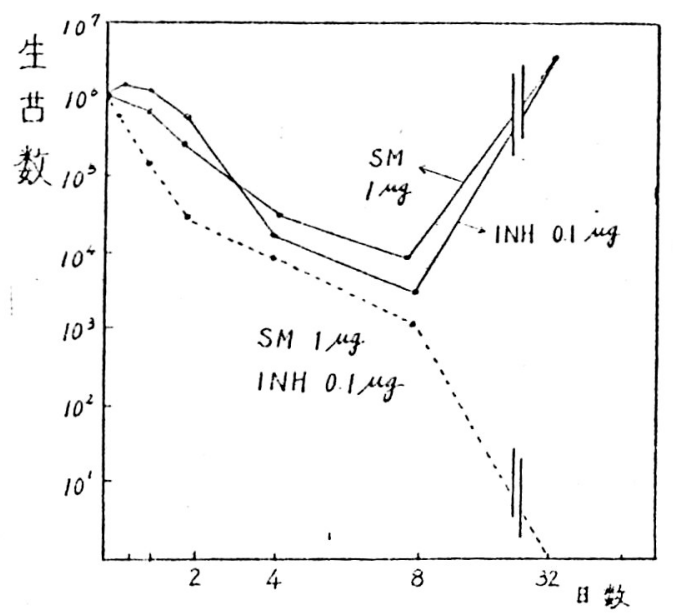

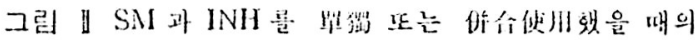

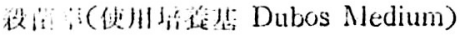

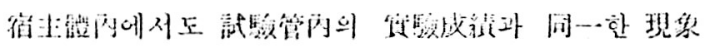

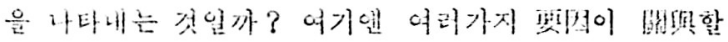

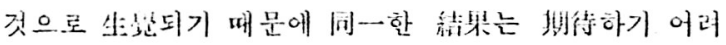

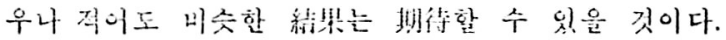

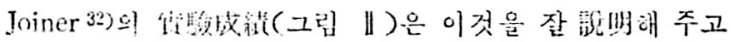
있다.

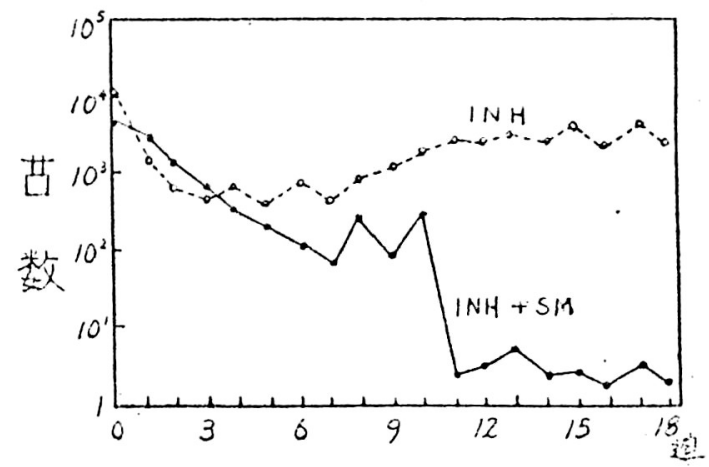

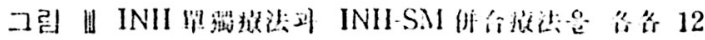

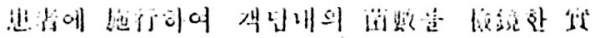
政拄.

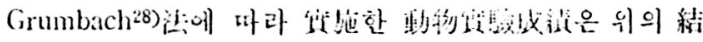

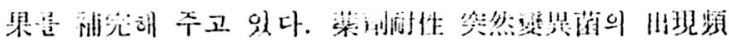

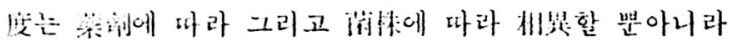

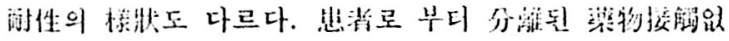

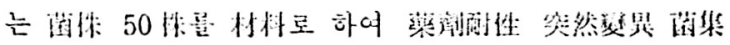

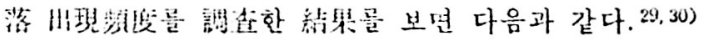

de I Titration pattern of normal strain of human tubercle bacilli according to the inoculation

\begin{tabular}{|c|c|c|c|}
\hline 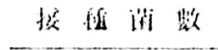 & $10^{6}$ & $10^{4}$ & $10^{2}$ \\
\hline Cotnrols & \multicolumn{3}{|c|}{ 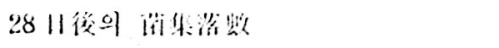 } \\
\hline \multicolumn{4}{|l|}{$\operatorname{INH}(\mu \mathrm{g} / \mathrm{ml})$} \\
\hline 0.05 & $20 \sim 40,000$ & $0 \sim .100$ & $0 \sim .1$ \\
\hline 0.1 & $0 \sim 200$ & $0 \sim 2$ & 0 \\
\hline 0.2 & $0 \sim 40$ & 0 & 0 \\
\hline 0.5 & $0 \sim 30$ & 0 & 0 \\
\hline 1 & $0 \sim 10$ & 0 & 0 \\
\hline 5 & $0 \sim 10$ & 0 & 0 \\
\hline 10 & $0 \sim 10$ & 0 & 0 \\
\hline \multicolumn{4}{|l|}{$\mathrm{SM}(\mu \mathrm{g} / \mathrm{ml})$} \\
\hline 1 & $1000 \sim 200,000$ & $10 \sim 2,000$ & $0 \sim 20$ \\
\hline 2 & $0 \sim 10,000$ & $0 \sim 100$ & $0 \sim 2$ \\
\hline 4 & $0 \sim 100$ & $0 \sim 1$ & 0 \\
\hline 10 & $0 \sim 10$ & 0 & 0 \\
\hline 100 & $0 \sim 1$ & 0 & 0 \\
\hline 1000 & $0 \sim 0.1$ & 0 & 0 \\
\hline \multicolumn{4}{|l|}{$\operatorname{PAS}(\mu g / m l)$} \\
\hline 0.12 & $0 \sim 200,000$ & $0 \sim 2,000$ & $-0-20$ \\
\hline 0.25 & $0 \sim 4,000$ & $0 \sim 40$ & 0 \\
\hline 0.5 & $0 \sim 40$ & 0 & 0 \\
\hline 1 & $0 \sim 40$ & 0 & 0 \\
\hline 10 & $0 \sim 1$ & 0 & 0 \\
\hline
\end{tabular}

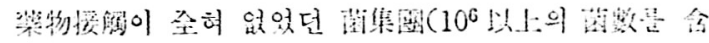

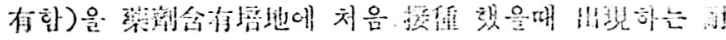

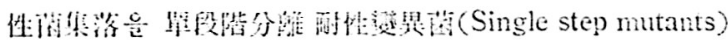

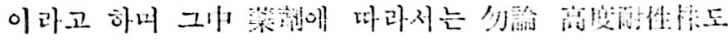

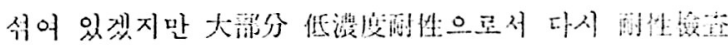

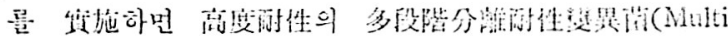

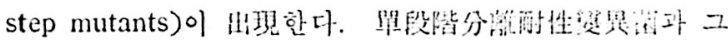

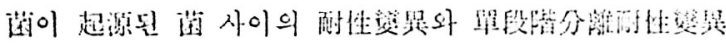

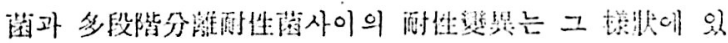

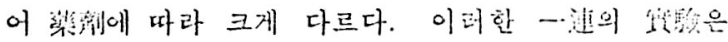
Tsukamura ${ }^{19)}$ 에 \{ \{챠해 이루엉 졌다.

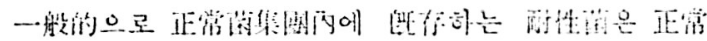

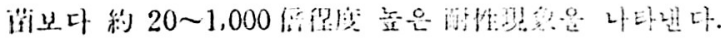

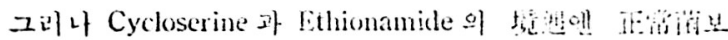

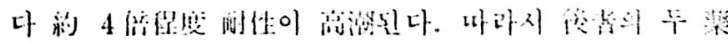

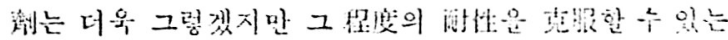

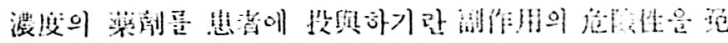

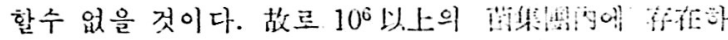

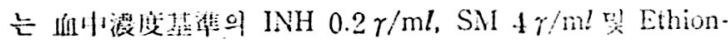

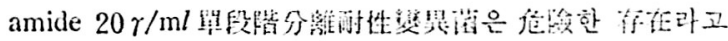
보겠다.

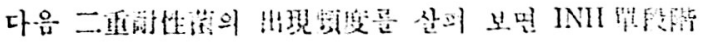


FUAf 서의

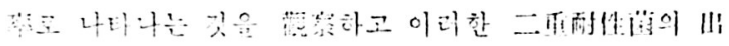

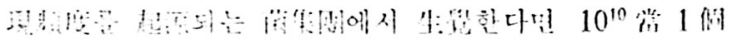

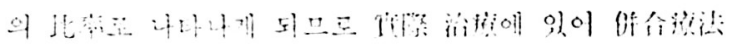

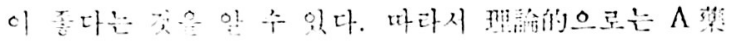

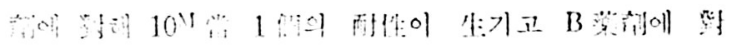

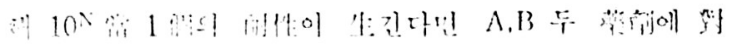

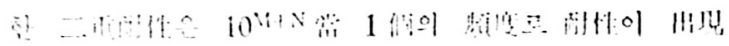

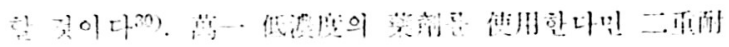

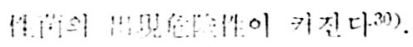

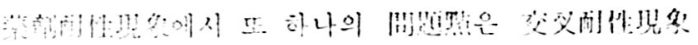

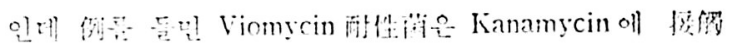
하어 왛있어소 Kanamycin

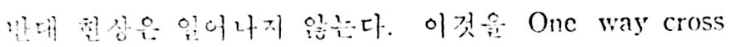

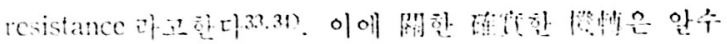

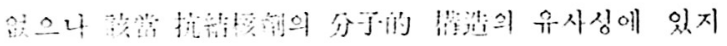

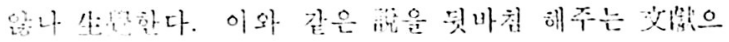

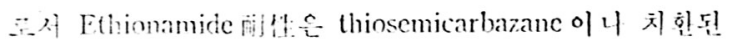

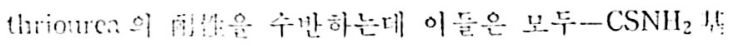

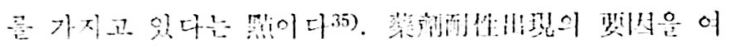

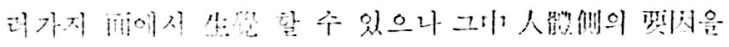

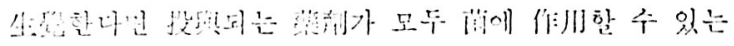

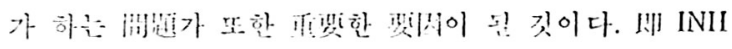

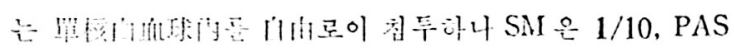

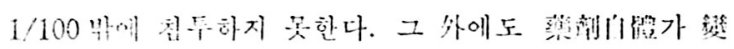

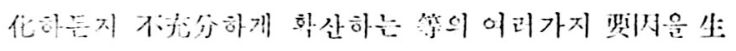
판한 수 의아.

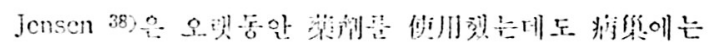

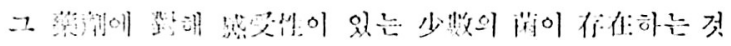

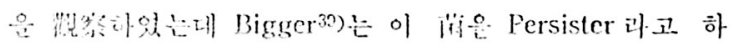

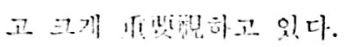

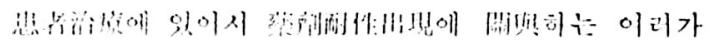

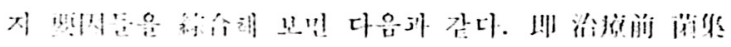

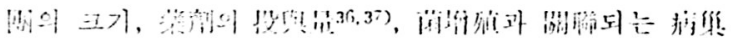

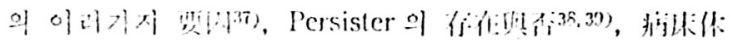

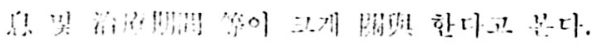

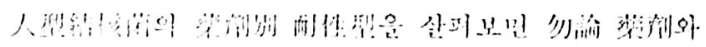

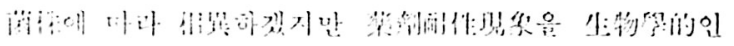

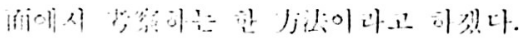

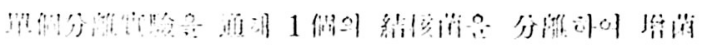

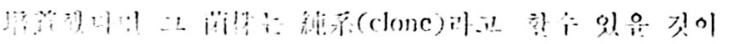

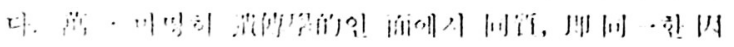

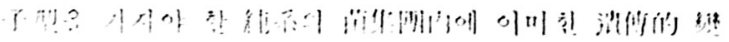

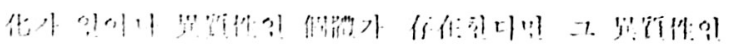

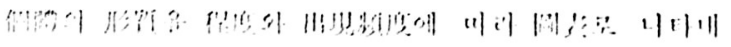

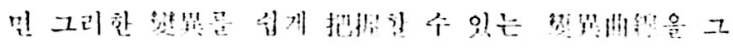

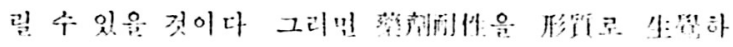

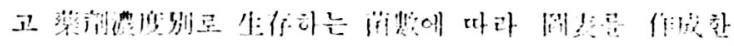

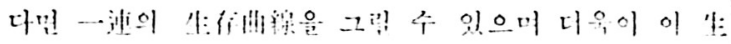

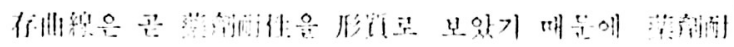

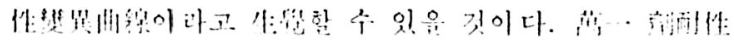

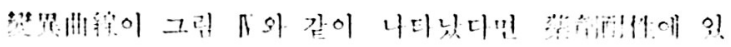

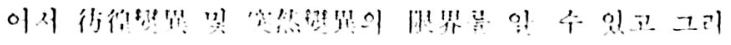

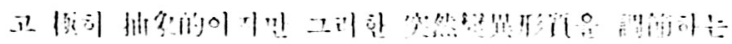

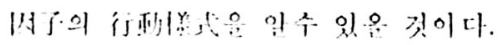

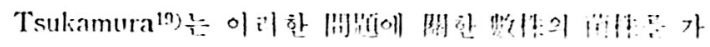

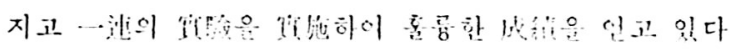

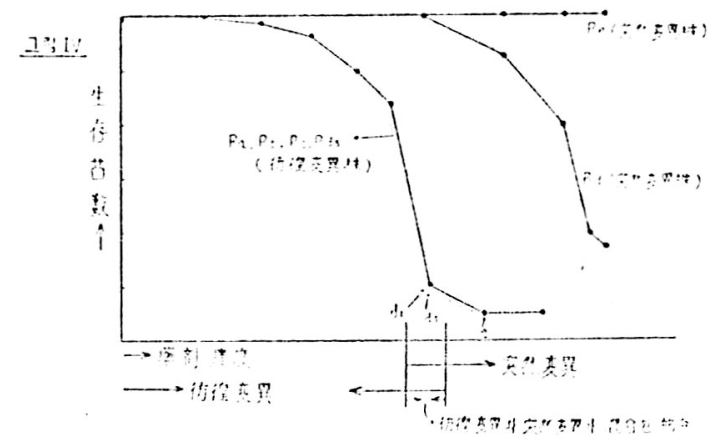

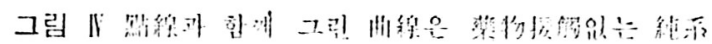

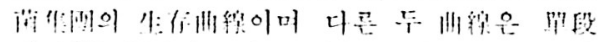

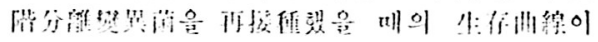
다.

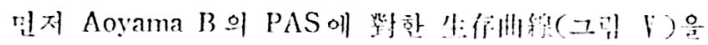
보면 f f

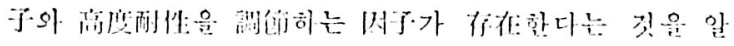

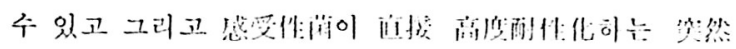

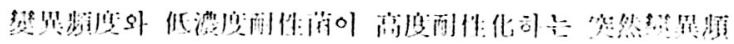

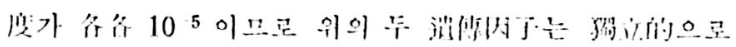
行切한다는 깃율 안 수 있다.

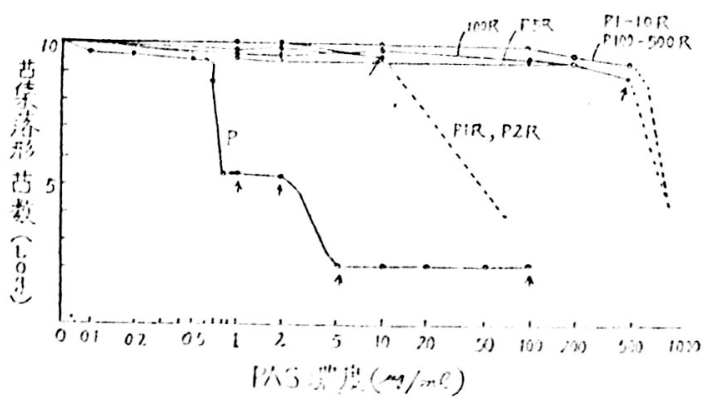

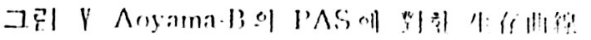




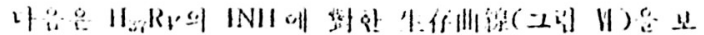

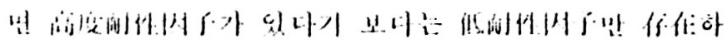

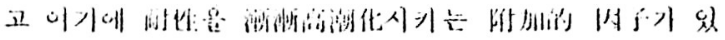

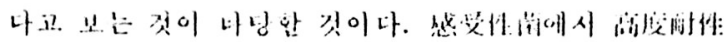
化of:

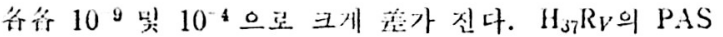
및 SM 的推도 이와 비솟하다.

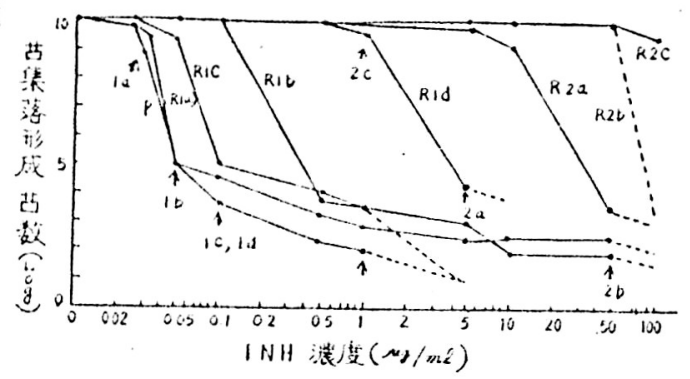

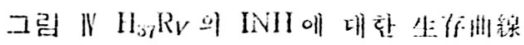

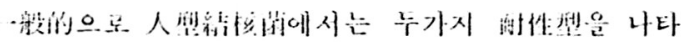

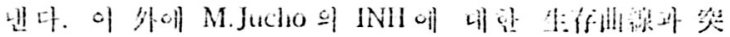

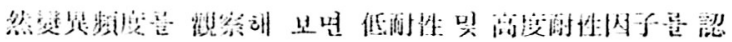

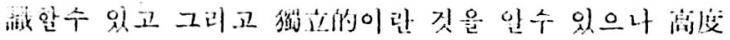

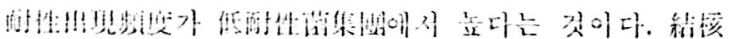

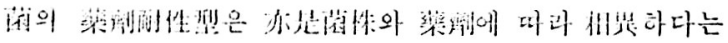
것을 안수 딨다.

\section{N 結論}

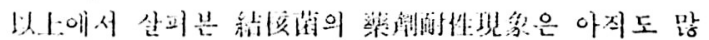

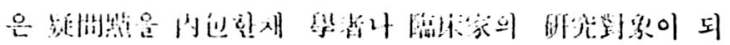

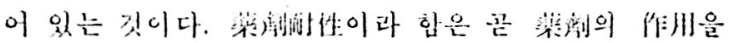

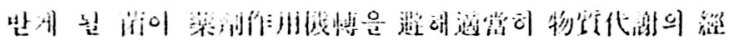

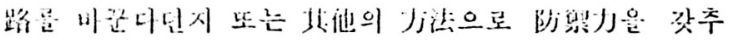

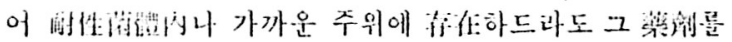

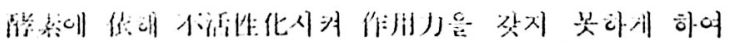

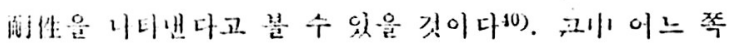

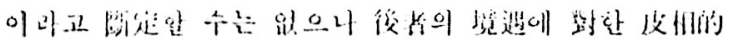

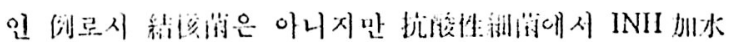

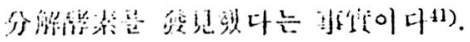

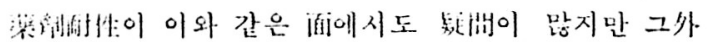

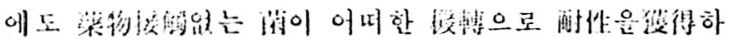

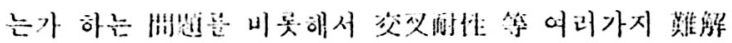
한 成题둘이 엉 있다.

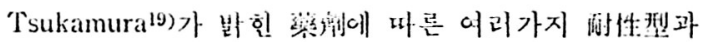

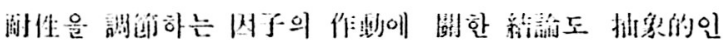

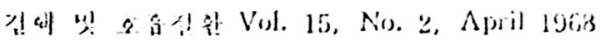

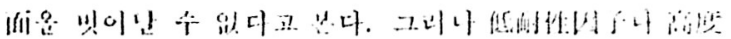

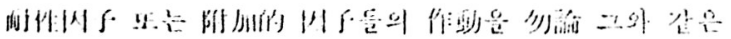

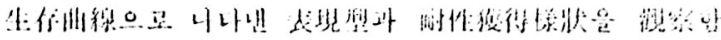

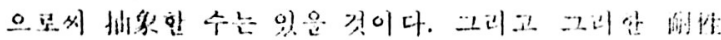

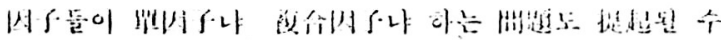

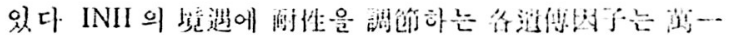

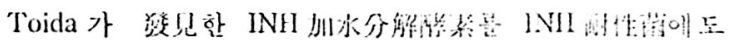

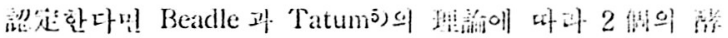

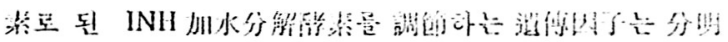

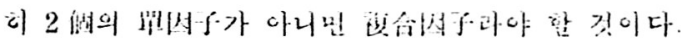

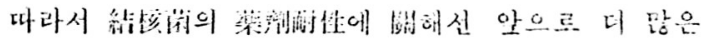
醉나 이루어저야 할 짓이다.

\section{References:}

1) Feldman, W.II, and Hinshaw, H.C: Proc. Mayoclin. $19.44,19,593$.

2) Bernheim, F: J. Bact., 19+1, +1, 387

3) Lehmann, J.: Lancel, 1946, 1. It

4) Fox, H.II.: J. chem. Eiduc. 1952, 17, $556 \ldots$

5) Sinnott,E.W., et al: Principles of Genetics, 5 thed. New York: McCraw-Iill, 195s

6) Gardner, E.J.: Principles of Genetics, New York, John Wiley und sons, 1961

7) Youmans, G.P., etal: Proc. Mayo Clin. 1640,21,126

8) Pyle, M.M.: Proc Mayoclin. 19.17, 22, to.5

9) Graessle, O.E.,et al.: J. Bact. 1949, 57, 459

10) Report: Medical Research Cuncil, Brit. med. J. $1952,1,1157$

11) D'Esope, N.D., et al.: Transaction th Conference on the chemotherapy of tuberculosis, Vetevans Administration, Army and Navy 1950, 59

12) Hobby, G.L.: Ari. Rev. Tubeve, 1152, 65, 771

13) Thomas, H.Z., et al.: Lancet, 1960, 2, 1185

14) Rist, N.: Advanc. Tulerc. Res. 1960, 10, 69

15) Hobby, G.L.: Ans. Rev. Tuberc, 1962, 56, 839

16) Mitchson. D.A.: Am. Rev. Thberc, 1554, 69:640

17) Thibier, R., et al: Rev. Tuberc. et Pnourol., paris $1900,2 t, 831$

18) Chicou, J, et al.: Rev. Tuberc et, Pueumal paris $1361,25,1031$

19) Tsukamura, M.: Jap. J. Tuberc. 1961, 9, 43

20) Hsie, J., etal. Am. Rev Tuberc, 1950, 62, 236

21) Ushiba, D.: Am. Rev. Tuterc, 11357, 75, s+1

22) Szybalski, W.: An. Reu. Tulerc, 1952, 65, 769.

23) Tsukamura, M., et al.: Am. Rev. Tuberc, 1955, 77, 519 


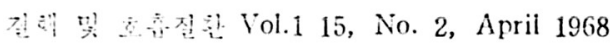

24) Verian, I)., ct al.: Am. Rev. Tuberc, 1950, 61, 483

25) Tsukamura, if., et al.: Kcklkuku, 1956, 31, 107.

26) Tsukamura, Mi. ct al.: Chemothcrafy, 1956, 4, 248.

27) Mackaness. G.B3.: Seminaire, Centre Inlcriational dc L'Enfance, paris p. 164, 1952. London;Lange, Aaxuell and Springer.

28) Rist $\mathrm{N}$. et al.: Tuberc ot Pncumol., paris, 1958, $22, \quad 29$.

29) Canctt. G. et al.: Amm. Inst. postcur, 1949, 77,611.

30) Grossct, 1., et al.: Ann. Inst. pasteur 1962, 103, 16.3.

31) Rist. $\backslash$. and Grumbach, I.: Rcv. Tuberce ct Pncumol., faris $1952.15,665$.
32) Joincr, C.L., et al.: Lancet et Pncumol., paris 1952. $2,84.3$.

33) Steenken, W.: Amer. Rev. Tuberc. 1959, 79, 66.

34) Tsukamura, M., et al: Amer. Rev. Resp. Dis. 1962. 85,427 .

35) Rist,N., et al.: Amer. Rev. Tuberc. 1959, 79, 1.

36) Mandel, W., et al.:J. Clin. Invest. 1959, 38, 1356.

37) Canctti,G.: Ann. Inct. Pastcur. 1959, 97, 53.

38) Jensen, K. А.: Bull. Un. int. Tubere. 1952, 22, 17.

39) Bigger, J.W.: Lancet 1944, 2, 497.

40) Barry, V.C. : Chemotherapy of Tuberculosis, London, Butlcrworths, 1964, p. 208.

41) Toida, I.: Amer. Rev. Tuberc. 1962., 85, 720. 